\title{
Species composition of extant coccolithophores including twenty six new records from the southwest Pacific near New Zealand
}

F. Hoe Chang ${ }^{1 *}$ and Lisa Northcote ${ }^{2}$

\begin{abstract}
Background: Coccolithophores are one of the major components of marine phytoplankton and also one of the most prominent members of haptophyte algae. Studies of the extant coccolithophores started more than half a century ago in New Zealand waters, and with two exceptions, were limited to only a few relatively small areas close to shore. In this study the diversity of these 'calcium carbonate scale-producers' were updated from specimens collected in oceanic waters around the wider region of New Zealand.

Methods: Water samples collected from 156 stations on 10 voyages between 2009 and 2011 were filtered through Nuclepore polycarbonate membrane filters. Coccolithophores retained on these filters were identified using scanning electron microscopy.

Results: A total of 46 extant coccolithophore taxa were identified from 160 samples collected around New Zealand. The total number of coccolithophore taxa identified was greatest to the east (46), intermediate to the west (15) and least to the northeast and south (4 each) of New Zealand. These coccolithophores were classified into seven families in the four orders, with three families in incertae sedis, and one in a nannolith family. Forty two taxa were heterococcolithophores and four were holococcolithophopres.

Conclusion: Approximately $57 \%$ of the extant coccolithophores recorded were first-time records for the region. Even though Syracosphaera taxa generally occurred at low frequencies, they were the largest group and made up $c$. $31 \%$ of all extant coccolithophores recorded in this study. Our findings provide updated information on the species composition and biogeography of coccolithophores in the southwest Pacific near New Zealand.
\end{abstract}

Keywords: Emiliania huxleyi, Extant coccolithophores, New records, Syracosphaera pemmadiscus, Species composition

\section{Background}

Coccolithophores (Coccolithophyceae) are marine, unicellular, golden-brown haptophytes (Probert et al. 2007; Ruggiero et al. 2015). At some stage in their life cycle they produce very small calcium carbonatescales called coccoliths. This group has been a major producer of calcite in the open ocean since the late Jurassic (Hay 2004). These algae have gained

\footnotetext{
* Correspondence: h.chang@niwa.co.nz

${ }^{1}$ Biodiversity and Biosecurity Group, National Institute of Water \&

Atmospheric Research Ltd., P. O. Box 14-901, Kilbirnie, Wellington 6241, New Zealand

Full list of author information is available at the end of the article
}

increased attention as they play an important role in the global carbon cycle (e.g., Hiramatsu and Deckker 1997; Baumann et al. 2000) and possibly are also susceptible to ocean acidification as are other calcifiers (e.g., Doney et al. 2009; Beaufort et al. 2011).

The most common form of coccolith is the heterococcolith (e.g., Cros and Fortuño 2002; Young et al. 2003). They are formed by crystal units of variable shape and size, and their biomineralisation occurs intracellularly (Manton and Leadale 1969). Another less common form is the holococcolith which is constructed from numerous minute crystallites that appear to get calcified extracellularly (e.g., Rowson et al. 1986; Kleijne 1991). Quite often both forms 
of coccolith of the same species are found in the same samples (e.g., Cros and Fortuño 2002; Young et al. 2003). The taxonomy of coccolithophores is primarily based on the morphology of the exquisite calcium carbonatecoccoliths of either heterococcolith or holococcolith form.

Living coccolithophores are distributed widely around the globe, from tropical to polar region (e.g., Thomsen 1981; Winter and Siesser 1994; Baumann et al. 2000; Findlay et al. 2005). Their biogeography has been studied most extensively in Mediterranean Sea, Indian, Pacific and Atlantic Oceans (e.g., Okada and McIntyre 1977; Kleijne 1993; Cros and Fortuño 2002; Young et al. 2003; Tyrrel and Merico 2004; Wang et al. 2012), and in relatively recent times in high latitudes of the Southern Ocean (e.g., Nishida 1986; Eynaud et al. 1999; Findlay and Giraudeau 2000; Saavedra-Pellitero et al. 2014).

In New Zealand, initial studies were limited to coccoliths collected from deep sediments over the Challenger Plateau (Murray and Renard 1891) and also in other parts of the New Zealand region (Edwards 1968, 1982; McIntyre et al. 1970; Burns 1973, 1975). Studies of living coccolithophores started more than half a century ago, however, were limited to several local areas near New Zealand (Cassie 1961; Burns 1977; Rhodes et al. 1993, 1994). The most extensive survey was conducted by Norris (1961) along a transect from northeast New Zealand to Tonga. Samples collected from this voyage were examined using light microscopy and records were not illustrated. Very recently a survey carried out in the Pacific sector of the Southern Ocean by Saavedra-Pellitero et al. (2014) examined some coccolithophore samples collected from the Subtropical Front (STF) and Subantarctic (SA) waters, east of New Zealand. But this study was limited to three sites in STF and SA waters in a summer. Up until now, no study has been conducted on species composition of extant coccolithophores in oceanic waters around the wider region of New Zealand.

The present study is a component of New Zealand research into the impacts of ocean acidification on pelagic plankton. The aims of this study were to determine the species composition of extant coccolithophores and to update the diversity (checklist) and distribution of this group including the wider region of the southwest Pacific near New Zealand. As a substantial number of taxa were new records, SEM images of all taxa are presented here. Cell abundance in relation to environmental variables is not included in this study but will be reported separately elsewhere (Law et al, manuscript in preparation).

\section{Methods}

\section{Coccolithophore samples}

A total of ten surveys were conducted aboard the RV's Tangaroa and Kaharoa from January 2009 to February 2012 in the southwest Pacific Ocean near
New Zealand (Fig. 1, Table 1). Seven of these surveys were conducted in spring, summer and autumn, in the subtropical (ST), STF and SA waters over the Chatham Rise and off Kaikoura coast, east of New Zealand (Fig. 1). The remaining three surveys were conducted on the northeast, west (along two transects in the Tasman Sea), and south of New Zealand. A combined total of 160 coccolithophore samples were collected from 156 stations around New Zealand (Fig. 1).

On each survey discrete water samples were collected from the upper $10 \mathrm{~m}$ using a 10-litre Niskin bottles mounted on a CTD rosette system. Immediately, 1.5 to 3 l water was filtered through a $47 \mathrm{~mm}$ diameter (0.8 $\mu \mathrm{m}$ pore size) Nuclepore Polycarbonate Track-Etch membrane filter (Whatman 111109). To minimise mechanical disruption of coccospheres, a vacuum of less than $100 \mathrm{~mm} \mathrm{Hg}$ (low pressure) was applied below the filter with an electrical vacuum pump. These filters were individually placed in a labelled plastic Millipore petri dish (PF10266, $47 \mathrm{~mm}$ diameter) and air dried. The filters were then stored in a sealed plastic storage container with desiccant until analysis.

\section{Scanning electron microscope}

Using a cork borer (10 $\mathrm{mm}$ in diameter) a small circular piece was cut out of the $47 \mathrm{~mm}$ Nuclepore polycarbonate membrane filter and mounted on JEOL $12 \times 10 \mathrm{~mm}$ aluminium slug using double-sided adhesive tape. These specimens were then coated with either platinum or carbon (15 nm thick) and examined with either a JEOL JSM-5300LV (Tokyo, Japan) or Quanta 450 (Oregon, U.S.A.) scanning electron microscopes (SEM) $(20 \mathrm{kV})$ as described in Chang (2013). For more than half of samples, high resolution images were further taken using a JEOL JSM6500F FEG-SEM (10 kV) (Tokyo, Japan). The entire coated, cut-out membrane filter was carefully examined using either JEOL or Quanta SEMs. The diameter/ length of coccospheres and coccoliths were measured using individual scales on the SEM images.

\section{Species identification}

Taxonomic identification employed the work of Okada and McIntyre (1977), Hallegraeff (1984, 2010), Kleijne (1992, 1993), Winter and Siesser (1994), Cros and Fortuño (2002), Young et al. (2003), Kleijne and Cros (2009) and Frada et al. (2010). The species list used here was based on that of Young et al. (2003) and Jordan et al. (2004).

\section{Results}

Species composition

A total of 46 extant coccolithophore taxa, two of which are represented by both hetero- and holococcolith forms, were identified from 160 samples collected from 


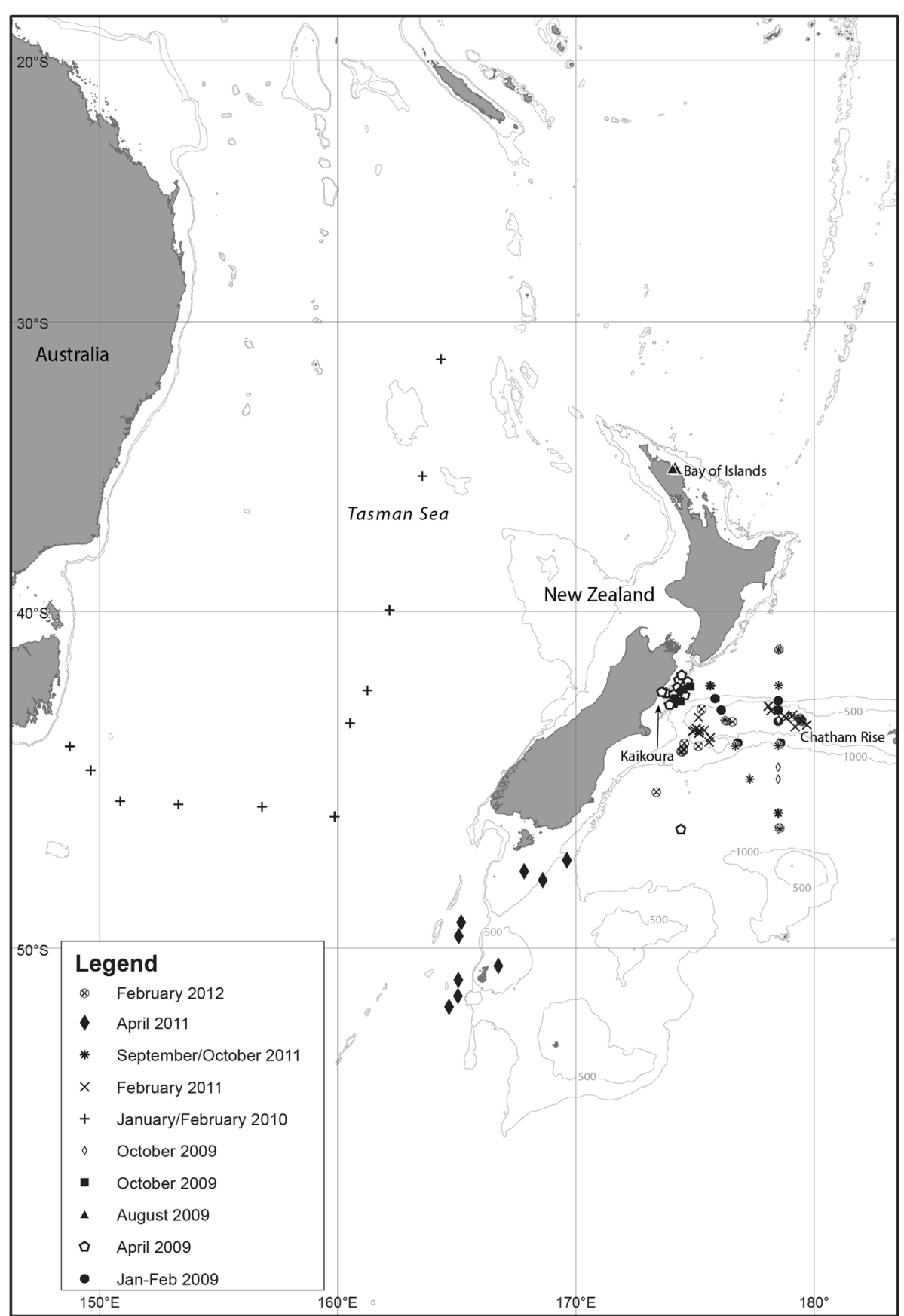

Fig. 1 Map of the study areas showing sampling stations around New Zealand

156 stations of ten surveys between 2009 and 2012 (Table 2; Figs. 2, 3 and 4). Forty two taxa were robust heterococcolithophores and 35 of them were placed in 7 families of the four orders, Coccosphaerales, Isochrysidales, Syracosphaerales and Zygodiscales, with the rest placed in incertae sedis (4 families) and in the holococcolith group according to the scheme of Young et al. (2003) and Jordan et al. (2004).
Syracosphaerales was by far the largest group, with 23 species/ taxa recorded in three families, Calciosoleniaceae (2), Rhabdosphaeraceae (7) and Syracosphaeraceae (14) (Table 2). Isochrysidales was intermediate, with 7 taxa in the family Noëlaerhabdaceae. Both Coccosphaerales (two species in the Calcidiscaceae) and Zygodiscales (three species plus one holococcolith form in Helicosphaeraceae and one species in Pontosphaeraceae) were 
Table 1 Number of stations where water samples were collected from each voyage during the period from 2009 to 2012 near New Zealand

\begin{tabular}{lllll}
\hline & Voyage & Location & Date & $\begin{array}{l}\text { Number of } \\
\text { stations }\end{array}$ \\
\hline 1 & TAN0902 & Chatham Rise & 28-01-09 to 3-02-09 & 15 \\
2 & TAN0904 & Kaikoura & 21-04-09 to 30-04-09 & 21 \\
3 & TAM0908 & Kaikoura & 1-10-09 to 21-10-09 & 4 \\
4 & TAN0909 & Chatham Rise & 16-10-09 to 30-10-09 & 19 \\
5 & KAH0907 & Bay of Islands & 20-8-09 to 22-8-09 & 3 \\
6 & PINTS & Tasman Sea & 31-01-10 to 15-02-10 & 36 \\
7 & TAN1102 & Chatham Rise & 6-02-11 to 11-02-11 & 16 \\
8 & TAN1107 & Chatham Rise & 28-09-11 to 1-10-11 & 9 \\
9 & TAN1106 & South of NZ & 19-04-11 to 29-04-11 & 8 \\
10 & TAN1203 & Chatham Rise & 5-02-12 to 28-02-12 & 25 \\
\hline
\end{tabular}

the least speciose. Six species (plus a holococcolith form of Coronosphaera mediterranea) were, however, placed in the three different groups of incertae sedis, with 4 species placed in the holococcolithophore group.

\section{Morphological characteristic}

As the majority of extant coccolithophores previously reported in New Zealand were examined using light microscopy and some with only low resolution SEM (e.g., Cassie 1961; Norris 1961; Burns 1973, 1975, 1977; Rhodes et al. 1993, 1994), the morphological characteristics of all 46 taxa recorded here were illustrated using mostly high resolution scanning electron microscopy. SEM images of 42 heterococcolithophores, including the two holococcolith forms [Helicosphaera carteri $\mathrm{HOL}=($ Syracolithus catilliferus (Kamptner 1937) Deflandre 1952) and Coronosphaera mediterranea HOL gracillima-type (Calyptrolithophora gracillima (Kamptner 1941) Heimdal in Heimdal and Gaarder 1980)], were displayed in Figs. 2a-t, $3 \mathrm{a}-\mathrm{t}$ and $4 \mathrm{a}-\mathrm{p}$, while the 4 holococcolithophores were illustrated in Fig. 4q-t.

Of the 46 taxa identified from samples collected between 2009 and 2012, 26 taxa/ forms were first-time records (c. $57 \%)$. Four of these first-time records were members of the order Isochrysidales, twelve were members of the order Syracosphaerales, three were members of the order Zygodiscales, another three were in the four families of insertae sedis, and four were holococcolithophores. In terms of size, coccoliths produced by the 26 first-time records of the New Zealand specimens (Table 3) were found to be generally reminiscent of those (with some variations) reported by Cros and Fortuño (2002) and Young et al. (2003). In the following only simple descriptions of the first-time records and a couple of new morphotypes (e.g., supercalcified and highly calcified) of Emiliania huxleyi (Lohmann 1902)
Hay and Mohler in Hay et al. 1967 found in the New Zealand region are presented here.

Gephyrocapsa muellerae Bréhéret 1978 (Fig. 2i), coccosphere spherical to subspherical $(5-6 \mu \mathrm{m})$. Elliptical placoliths (2.9-3.8 $\mu \mathrm{m}$ long) are similar in construction to those of Gephyrocapsa oceanica Kamptner 1943, with bridge at low angle to long axis; central area rather small.

Gephyrocapsa ornata Heimdal 1973 (Fig. 2k-l), coccosphere spherical to subspherical $(4-5 \mu \mathrm{m})$ (Table 2), coccoliths similar to G. ericsonii McIntyre and Bé 1967 (Fig. 2h) but with a conspicuous ring of spines around the central area and with much higher bridge of two diametrically opposite plates of varying shape. The bridge of G. ornata is at low angle to the long axis.

Reticulofenestra parvula (Okada and McIntyre 1977) Biekart 1989 (Fig. 2m) and R. parvula var. tecticentrum (Okada and McIntyre 1977) Jordan and Young 1990 (Fig. 2n), coccospheres $(4-6 \mu \mathrm{m})$ of both species/variety similar to Emiliania huxleyi (Fig. 2c-g; Table 2). Coccoliths of $R$. parvula differed from those of E. huxleyi in not having slits between distal shield elements while $R$. parvula var. tecticentrum differed from the latter species in having coccolith with over calcified central area.

Anacanthoica acanthos (Schiller 1925) Deflandre 1952 (=Acanthoica) (Fig. 2s), coccosphere ovoid (c. $6 \mu \mathrm{m}$ ), monomorphic with no spines (Table 2). Coccoliths elliptical $(1.9-2.1 \mu \mathrm{m})$ with relatively wide rim; ring of radial laths with central, wide, low protrusion (Table 3).

Cyrtosphaera aculeata (Kamptner 1941) Kleijne 1992 (=Acanthoica) (Fig. 2t), coccosphere $(8-10 \mu \mathrm{m})$ subspherical to elongate (Table 2). Coccoliths $(2.5-2.8 \mu \mathrm{m})$ varimorphic with rim somewhat bent upwards; central area with ring of radial laths (Table 3). Conical inner central area with protrusion ending in papilla; protrusion of some apical coccoliths modified into spine.

Palusphaera vandelii Lecal 1965 emend. Norris 1984 (Fig. 3b), coccosphere subspherical (without spines, 4.4$4.7 \mu \mathrm{m})$ (Table 2). Coccoliths with relatively long, distal spines and almost circular proxima disc $(1.5-2.0 \mu \mathrm{m})$ with smooth central area towards base of spine (Table 3).

Rhabdosphaera xiphos (Deflandre and Fert 1954) Norris 1984 (Fig. 3c-d), coccosphere subspherical (4.0$5.8 \mu \mathrm{m}$ ) and dimorphic (Table 2). There is a distinctive star pattern on distal face of circular body coccolith $(1.3-1.9 \mu \mathrm{m})$ with delicate spine and short collar at its base (Fig. 3d) (Table 3).

Syracosphaera anthos (Lohmann 1912) Janin 1987 (=Deutshlandia) (Fig. 3e-f), coccosphere subspherical $(12.3-15.3 \mu \mathrm{m})$ and dithecate. Body coccolith (4.5$5.8 \mu \mathrm{m})$ dimorphic, circum-flagellar coccolith with large spine (Tables 2 and 3).

Syracosphaera bannockii (Borsetti and Cati 1976) Cros et al. 2000 (Fig. 3g-h), coccosphere ovoid $(6.2-8.2 \mu \mathrm{m})$ and dithecate; body coccoliths $(1.7-2.8 \mu \mathrm{m})$ elliptical 
Table 2 List of extant coccolithophores recorded from 160 samples collected from 156 stations during 2009 and 2012: a) over the Chatham Rise and Kaikoura coast, east of New Zealand (NZ); b) in Bay of Plenty, northeast NZ; c) along two transects in the Tasman Sea, west of NZ; d) south of NZ

\begin{tabular}{|c|c|c|c|c|c|c|c|c|c|c|c|}
\hline \multirow[t]{2}{*}{ Taxa } & \multirow{2}{*}{$\begin{array}{l}\text { Figure } \\
\text { No. }\end{array}$} & \multirow{2}{*}{$\begin{array}{l}\text { Cell } \\
\text { size } \\
(\mu \mathrm{m})\end{array}$} & \multicolumn{4}{|l|}{ Chatham Rise } & \multicolumn{2}{|c|}{ Kaikoura } & \multirow{2}{*}{$\frac{\text { Bay of Plenty }}{\text { SP } 09}$} & \multirow{2}{*}{$\frac{\text { Tasman Sea }}{\text { SU } 10}$} & \multirow{2}{*}{$\frac{\text { South of NZ }}{A U 11}$} \\
\hline & & & SP 09 SU 09 & SU 11 & AU 11 & SU 12 & SP 09 & AU 09 & & & \\
\hline
\end{tabular}

Heterococcolith Group

Order Coccosphaerales

Family Calcidiscaceae

Calcidiscus leptoporus

Umbilicosphaera hulburtiana

$\begin{array}{lll}2 \mathrm{~A} & 10-15+ \\ 2 \mathrm{~B} & 5-6 & -\end{array}$

Order Isochrysidales

Family Noëlaerhabdaceae

$\begin{array}{llll}\text { Emiliania huxleyi } & 2 \mathrm{C}-\mathrm{G} & 3-7 & +^{*} \\ \text { Gephyrocapsa ericsonii } & 2 \mathrm{H} & 4-5 & + \\ \text { Gephyrocapsa muellerae } & 2 \mathrm{l} & 5-6 & - \\ \text { Gephyrocapsa oceanica } & 2 \mathrm{~J} & 8-10 & + \\ \text { Gephyrocapsa ornata } & 2 \mathrm{~K}-\mathrm{L} & 4-5 & - \\ \text { Reticulofenestra parvula } & 2 \mathrm{M} & 4-6 & - \\ \text { R. parvula var. tecticentrum } & 2 \mathrm{~N} & 4-6 & -\end{array}$

Order Syracosphaerales

Family Calciosoleniaceae

\begin{tabular}{|c|c|c|c|}
\hline Calciosolenia brasiliensis & $3 \mathrm{G}-\mathrm{H}$ & $6-8$ & - \\
\hline Calciosolenia murrayi & 31 & $11-13$ & - \\
\hline Family Rhabdosphaeraceae & $3 J-K$ & $6-7$ & - \\
\hline Acanthoica quattrospina & $3 L-M$ & $6-9$ & - \\
\hline Algirosphaera robusta & $3 \mathrm{~N}$ & $6-7$ & - \\
\hline Anacanthoica acanthos & 30 & $5-6$ & - \\
\hline Cyrtosphaera aculeata & $3 P-Q$ & c. 11 & - \\
\hline Discosphaera tubifera & $3 R$ & c. 7 & - \\
\hline Palusphaera vandelii & $3 S$ & $6-7$ & - \\
\hline Rhabdosphaera xiphos & зТ & $7-8$ & - \\
\hline Family Syracosphaeraceae & $4 \mathrm{~A}$ & $10-12$ & + \\
\hline Syracosphaera anthos & $4 B-C$ & c. 9 & - \\
\hline Syracosphaera bannockii & $4 \mathrm{D}$ & n.d. & - \\
\hline \multicolumn{4}{|l|}{ Syracosphaera corolla } \\
\hline Syracosphaera leptolepis & $4 \mathrm{E}$ & $12-15$ & - \\
\hline Syracosphaera molischii & $3 \mathrm{G}-\mathrm{H}$ & $6-8$ & - \\
\hline Syracosphaera nana & 31 & $11-13$ & - \\
\hline Syracosphaera nodosa & $3 J-K$ & $6-7$ & - \\
\hline S. nodosa aff. S. sp. type 2 & $3 L-M$ & $6-9$ & - \\
\hline Syracosphaera cf. orbiculus & $3 \mathrm{~N}$ & $6-7$ & - \\
\hline Syracosphaera ossa & 30 & $5-6$ & - \\
\hline Syracosphaera pemmadiscus & $3 P-Q$ & c. 11 & - \\
\hline Syracosphaera pulchra & $3 R$ & c. 7 & - \\
\hline Syracosphaera serrata & $3 S$ & $6-7$ & - \\
\hline Syracosphaera tumularis & 3T & $7-8$ & - \\
\hline
\end{tabular}


Table 2 List of extant coccolithophores recorded from 160 samples collected from 156 stations during 2009 and 2012: a) over the Chatham Rise and Kaikoura coast, east of New Zealand (NZ); b) in Bay of Plenty, northeast NZ; c) along two transects in the Tasman Sea, west of NZ; d) south of NZ (Continued)

\begin{tabular}{|c|c|c|c|c|c|c|c|c|c|c|c|c|}
\hline Genus incertae sedis & $4 \mathrm{~A}$ & $10-12$ & + & + & - & + & + & - & + & - & + & - \\
\hline Coronosphaera mediterranea & $4 \mathrm{~B}-\mathrm{C}$ & c. 9 & - & - & - & - & - & - & - & - & - & - \\
\hline \multicolumn{13}{|l|}{ Coronosphaera mediterranea } \\
\hline HOL gracillima-type & $4 \mathrm{~F}$ & n.d. & - & - & - & - & - & - & - & - & + & - \\
\hline \multicolumn{13}{|l|}{ Order Zygodiscales } \\
\hline \multicolumn{13}{|l|}{ Family Helicosphaeraceae } \\
\hline Helicosphaera carteri & $4 G$ & $17-19$ & - & + & - & - & + & - & + & - & + & - \\
\hline Helicosphaera carteri HOL & $4 \mathrm{H}$ & c. 12 & - & + & - & - & - & - & - & - & - & - \\
\hline Helicosphaera hyalina & 41 & c. 12 & - & + & - & - & - & - & - & - & - & - \\
\hline Helicosphaera wallichii & 4J & c. 15 & - & + & - & - & - & - & - & - & - & - \\
\hline \multicolumn{13}{|l|}{ Family Pontosphaeraceae } \\
\hline Scyphosphaera apsteinii & $4 \mathrm{~K}$ & c. 26 & - & + & - & - & - & - & + & - & - & - \\
\hline \multicolumn{13}{|l|}{ Coccolith families incertae sedis } \\
\hline \multicolumn{13}{|l|}{ Family Alisphaeraceae } \\
\hline Alisphaera pinnigera & $4 \mathrm{~L}$ & c. 7 & - & - & - & + & - & - & + & - & - & - \\
\hline Polycrater galapagensis & $4 \mathrm{M}$ & c. 8 & - & + & - & - & - & - & - & - & - & - \\
\hline \multicolumn{13}{|l|}{ Family Papposphaeraceae } \\
\hline Papposphaera lepida & $4 \mathrm{~N}$ & c. 5 & - & + & - & + & - & - & - & - & - & - \\
\hline \multicolumn{13}{|l|}{ Family Umbellosphaeraceae } \\
\hline Umbellosphaera tenuis Type ॥ & 40 & $8-14$ & + & + & + & + & - & - & - & - & + & + \\
\hline \multicolumn{13}{|l|}{ Nannolith family incertae sedis } \\
\hline \multicolumn{13}{|l|}{ Family Braarudosphaeraceae } \\
\hline Braarudosphaera bigelowii & $4 \mathrm{P}$ & c. 5-6 & - & + & - & - & - & - & - & - & - & - \\
\hline \multicolumn{13}{|l|}{ Holococcolith Group } \\
\hline Corisphaera gracilis & $4 Q$ & c. 5 & - & + & - & + & - & - & - & - & - & - \\
\hline Holococcolithophora sphaeroidea & $4 \mathrm{R}$ & $8-10$ & - & + & - & - & - & - & - & - & - & - \\
\hline Homozygosphaera arethusae & $4 S$ & $8-12$ & - & + & - & - & - & - & - & - & - & - \\
\hline Poricalyptra aurisinae & $4 \mathrm{~T}$ & c. 8 & - & + & - & - & - & - & - & - & - & - \\
\hline
\end{tabular}

${ }^{5}$ Cell size is a measurement of either the diameter or length of cell in $\mu \mathrm{m} ;{ }^{*}$ Emiliania huxleyi blooms confirmed by the MODIS sensor on NASA'S satellite (NASA Earth Observatory 2009, 2011); ' + ' = present, ' $-'=$ absent; SP 09=Spring 2009; SU 09=Summer 2009; AU 09=Autumn 2009; SU $11=$ Summer 2011; SU 10=Summer 2010; AU 11 = Autumn 2011; SU 12 = Summer 2012

with tube-like structure; circum-flagellar coccoliths with pointed spine, sometimes slightly curved (Tables 2 and 3).

Syracosphaera leptolepis Kleijne and Cros 2009 (Fig. 3j-k), coccosphere subspherical (c. $7.0 \mu \mathrm{m}$ ); body coccoliths $(1.8-2.0 \mu \mathrm{m})$ broadly elliptical dishes with slight distal edge and straight wall; central area slightly vaulted with slightly raised, inner central structure. Exothecal planoliths circular discs, central part consisting of dextrally oblique elements (Fig. 3k); loosely attached to the coccospheres (Tables 2 and 3).

Syracosphaera nodosa Kamptner 1941 (Fig. 3o), coccosphere ovoid $(6.2-6.6 \mu \mathrm{m})$ and dithecate. Body coccolith elliptical $(1.3-2.7 \mu \mathrm{m})$, wall relatively deep but with no distal flange and with characteristic vertical ribs on outer surface of wall (Tables 2 and 3). Circum-flagellar coccolith has strong spine, with sheath-like structure which covers about $80 \%$ of proximal part of the spine.

Syracosphaera nodosa aff. S. sp. type 2 of Kleijne 1993 (Fig. 3p-q), coccosphere almost spherical (c. $11 \mu \mathrm{m}$ ), and dithecate (Table 2). Thin and subcircular outer coccoliths form complete outer layer over body coccoliths. Central area of elliptical body coccolith $(2.8-2.9 \mu \mathrm{m})$ with radial laths and elongated mound as central connecting structure; thin, subcircular exothecal planoliths loosely attached to coccosphere (Fig. 3q; Table 3).

Syracosphaera cf. orbiculus Okada and McIntyre 1977 (Fig. 3r), coccosphere subspherical (c. $7.0 \mu \mathrm{m})$. Body coccoliths $(2.2-2.6 \mu \mathrm{m})$ with relatively thin smooth wall (Tables 2 and 3); central area with well-developed connecting external ring and flat, elongated internal 

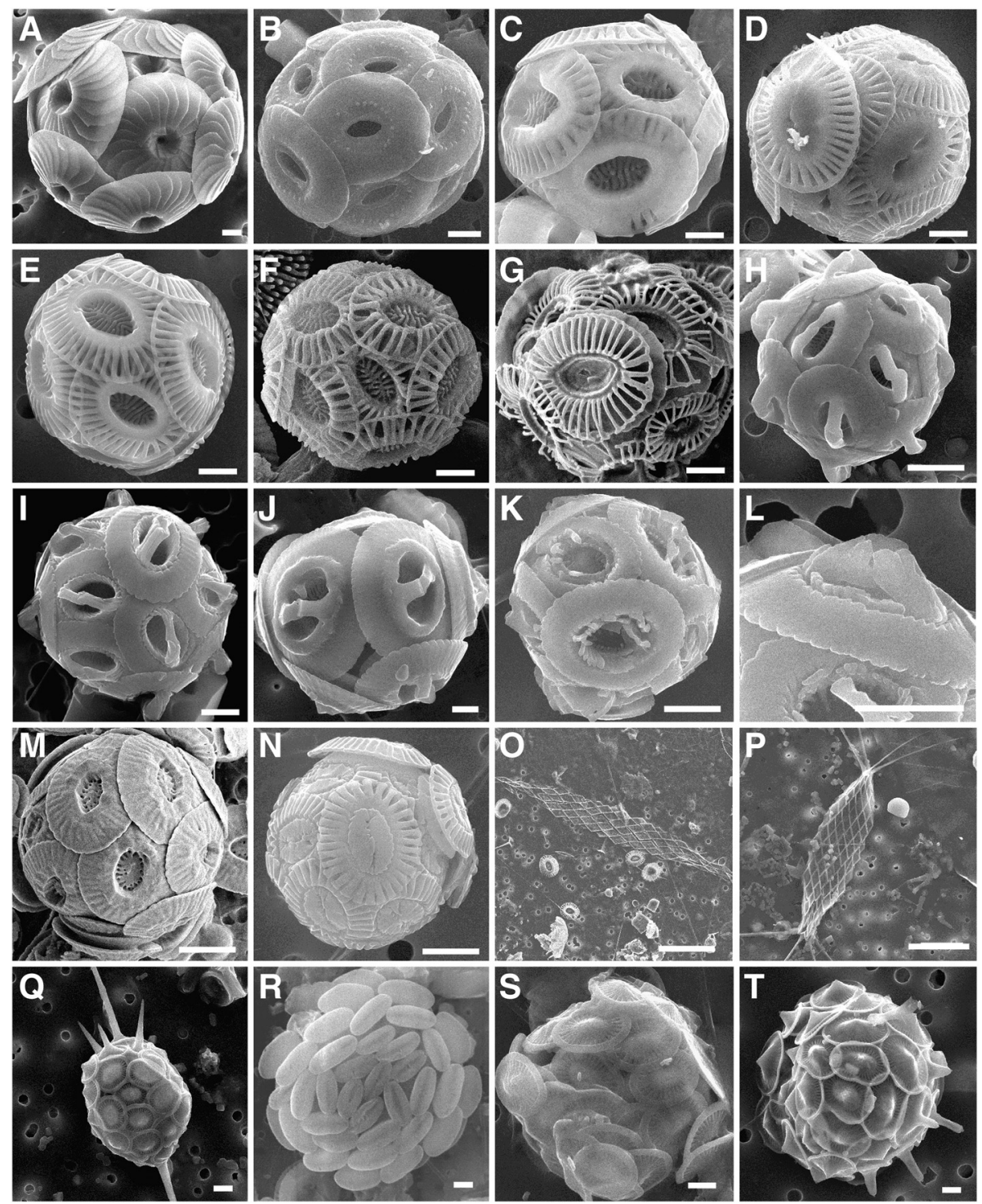

Fig. 2 Heterococcolith and holococcolith forms: (a) Calcidiscus leptoporus; (b) Umbilicosphaera hulburtiana; (c-g) Emiliania huxleyi, showing five different morphotypes (c, supercalcified; $\mathbf{d}$, over-calcified; e, type A; $\mathbf{f}$, type B; $\mathbf{g}$; type C); (h) Gephyrocapsa ericsonii; (i) Gephyrocapsa muelerae; (j) Gephyrocapsa oceanica; (k) Gephyrocapsa ornata; (I) Coccolith of G. ornata; (m) Reticulofenestra parvula; (n) Reticulofenestra parvula var. tecticentrum; (o) Calciosolenia brasiliensis; (p) Calciosolenia murrayi; (q) Acanthoica quattrrospina; (r) Algirosphaera robusta; (s) Anacanthoica acanthos; (t) Cyrtosphaera aculeata. Scale bars: a-n and $\mathbf{q}-\mathbf{t}, 1 \mu \mathrm{m} ; \mathbf{o}-\mathbf{p}, 10 \mu \mathrm{m}$

connecting structure. Circum-flagellar coccoliths with long and somewhat bent spine (Cros and Fortuño 2002).

Syracosphaera serrata Kleijne and Cros 2009 (Fig. 4b-c), coccosphere $(c .9 \mu \mathrm{m})$ dithecate (Table 2). Body coccoliths broadly elliptical $(2.1-2.2 \mu \mathrm{m})$ with irregular outline and low, thin flaring wall; central area made up of radial laths and a flat inner centre (Table 3). Exothecal coccoliths are wheel-like planoliths with serrate margin (Fig. 4c).

Coronosphaera mediterranea HOL gracillima-type (=Calyptrolithophora gracillima (Kamptner 1941) Heimdal in Heimdal and Gaarder 1980) (Fig. 4f), holococcolith form. Body coccoliths $(2.8-3.2 \mu \mathrm{m})$ have rounded distal protrusion with flared tube (Table 3 ), no flange, hexagonal mesh fabric without large pores; discontinuous rim formed from two rows of crystallites.

Helicosphaera carteri HOL [=Syracolithus catilliferus (Kamptner 1937) Deflandre, 1952 (=Syracosphaera)] (Fig. 4h), holococcolith form produced only small elliptical coccoliths $(2.4-2.7 \mu \mathrm{m})$ (Table 3$)$ with central pyramidal spine.

Helicosphaera hylina Gaarder 1970 (Fig. 4i) and H. wallichii (Lohmann 1902) Okada and McIntyre 1977 (Fig. 4j), coccospheres of the heterococcolith form of 

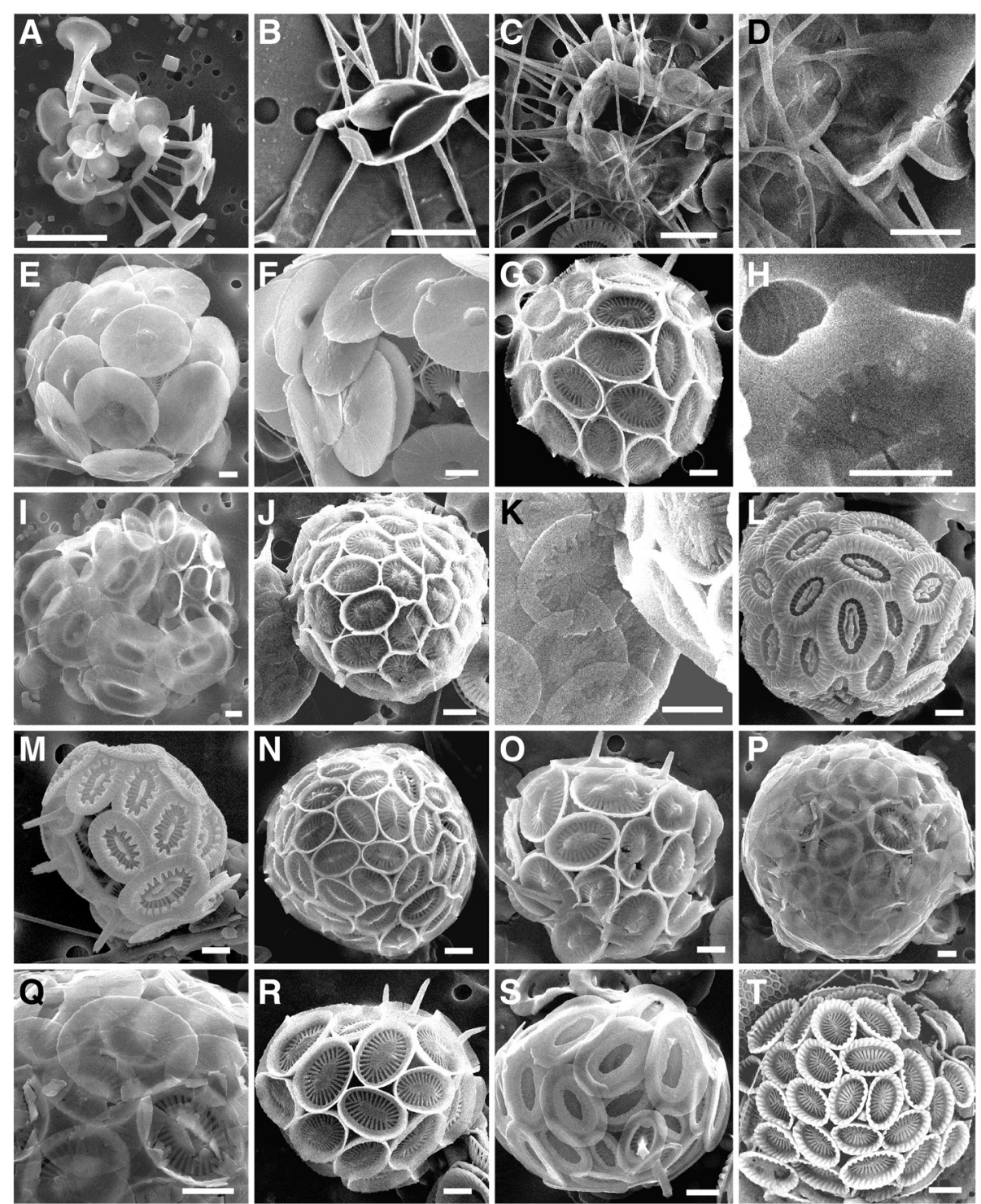

Fig. 3 Heterococcolith form: (a) Discosphaera tubifera; (b) Palusphaera vandelii; (c) Rhabdosphaera xiphos; (d) Coccoliths of R. xiphos, showing a distinctive star pattern on the distal surface; (e) Syracosphaera anthos; (f) Circum-flagellar coccoliths of S. anthos, showing a prominent spine; (g) Syracosphaera bannockii; (h) Exothecal coccolith of S. bannockii; (i) Syracosphaera corolla; (j) Syracosphaera leptolepis; (k) Exothecal coccoliths of S. leptolepis; (I) Syracosphaera molischii type 1; (m) S. molischii type 2; (N) Syracosphaera nana; (o) Syracosphaera nodosa; (p) Syracosphaera sp. aff. S. nodosa type 2 of Kleijne; (q) Thin circular exothecal coccoliths of S. sp. aff. S. nodosa type 2; (r) Syracosphaera cf. orbiculus; (s) Syracosphaera ossa; (t) Syracosphaera pemmadiscus. Scale bars: a-t, 1 mm

both species $(<15 \mu \mathrm{m})$ produced relatively large coccoliths $(5.3-6.0 \mu \mathrm{m}$ and $8.4-10 \mu \mathrm{m}$ respectively) (Tables 2 and 3) that wedged into one another by winged flanges, reminiscent of those of $H$. carteri (Wallich 1877) Kamptner 1954. There were, however, no slits in central area of $H$. hylina, while central area of $H$. wallichii has oblique twisted slits, rather than two inline slits as with H. carteri.

Alisphaera pinnigera Kleijne et al. 2002 (Fig. 4l), coccosphere $(c .7 \mu \mathrm{m})$ dimorphic. Central area of coccolith $(1.2-1.5 \mu \mathrm{m})$ has horizontal fissure (Tables 2 and 3).
Some coccoliths have either tooth-like or flat, triangularlike protrusion along their inner margin.

Polycrater galapagensis Manton and Oates 1980 (Fig. $4 \mathrm{~m})$, coccosphere $(15-16 \mu \mathrm{m})$ with numerous very small coccoliths $(0.6-0.7 \mu \mathrm{m})$ (Tables 2 and 3 ). Quadrate in plan-view and upside-down triangle-shaped in lateral view. Coccoliths of aragonite (Manton and Oates 1980).

Papposphaera lepida Tangen 1972 (Fig. 4n), cell spherical $(5-6 \mu \mathrm{m})$, diameter of coccosphere 14-15 $\mu \mathrm{m}$. Base of coccolith $(1.2-1.6 \mu \mathrm{m})$ subcircular to elliptical, central 

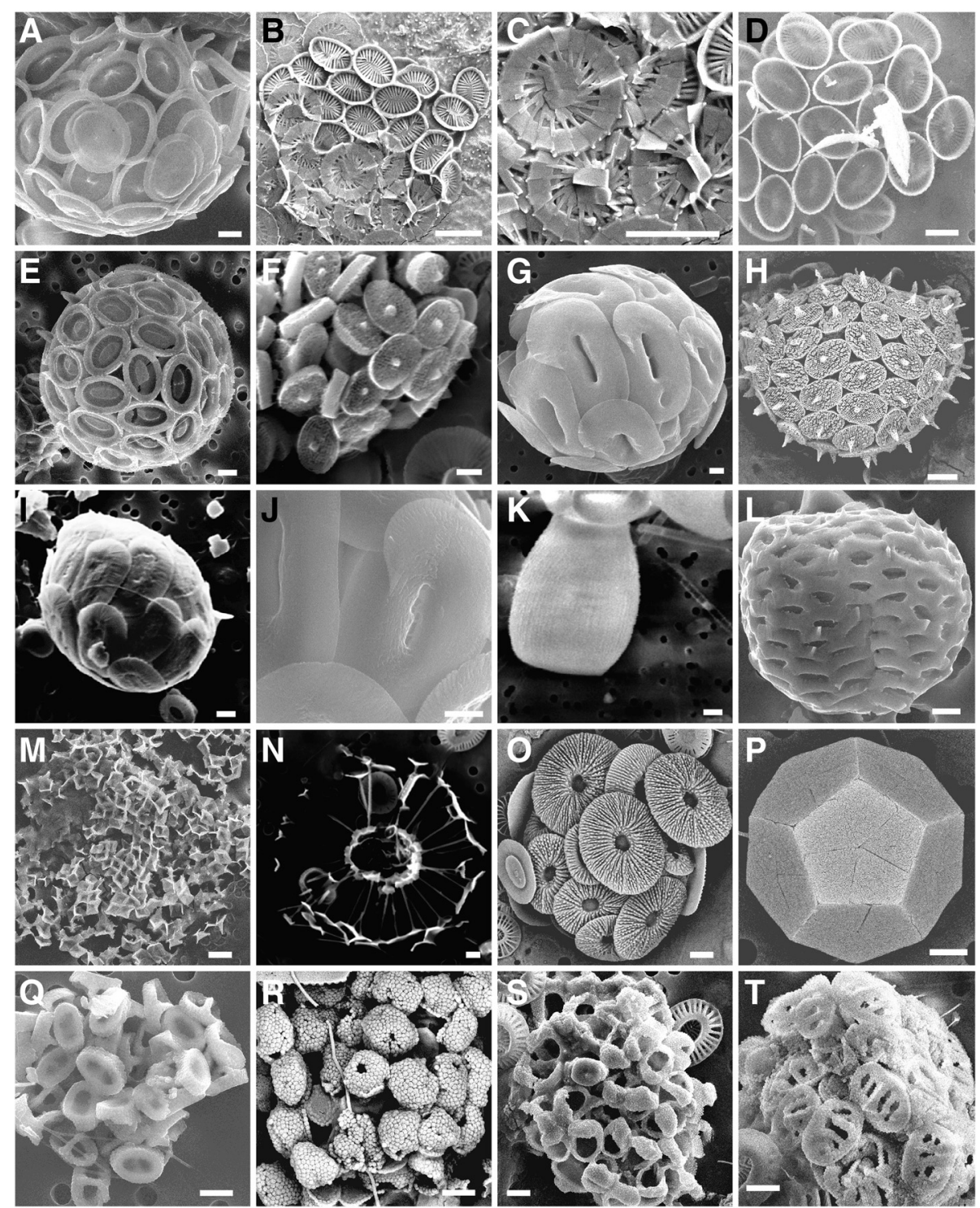

Fig. 4 Heterococcolith and holococcolith forms: (a) Syracosphaera pulchra; (b) Syracosphaera serrata; (c) Wheel-like exothecal planoliths of S. serrata; (d) Syracosphaera tumularis; (e) Coronosphaera mediterranea; (f) Coronosphaera mediterranea HOL gracillima-type; (g) Helicosphaera carteri; (h) Helicosphaera carteri HOL (= Syracolithus catilliferus) (i) Helicosphaera hylina; (j) Helicosphaera wallichii; (k) Scyphosphaera apsteinii; (I) Alisphaera pinnigera; (m) Polycrater galapagensis; (n) Papposphaera lepida; (o) Umbellosphaera tenuis type II; (p) Braarudosphaera bigelowii; (q) Corisphaera gracilis; (r) Holococcolithophora sphaeroidea; (s) Homozygosphaera arethusae; (t) Poricalyptra aurisinae. Scale bars: A-T, 1 um

area with long central stem which supports flat cone of four large elements giving rise to almost continuous outer layer of cell.

Corisphaera gracilis Kamptner 1937 (Fig. 4q), coccosphere subspherical $(c .5 \mu \mathrm{m})$; coccoliths $(1.2-1.5 \mu \mathrm{m})$ with low wall and transverse arched bridge across open distal end (Tables 2 and 3). Proximal end appears to be sealed by thin-layer of crystallites.

Holococcolithophora sphaeroidea (Schiller 1913) Jordan et al. 2004 (=Calyptrosphaera) (Fig. 4r), coccosphere ovoid (c. 10-12 $\mu \mathrm{m}$ long) (Table 2). Coccoliths conical with basal flange; distal end tapers abruptly into small projection. Microcrystallites irregularly arranged, separated by small perforations.

Homozygosphaera arethusae (Kamptner 1941) Kleijne 1991 (=Corisphaera) (Fig. 4s), coccosphere ovoid $(8-12 \mu \mathrm{m})$ (Table 2); coccoliths $(1.7-2.1 \mu \mathrm{m})$ have proximal tube and robust, arched distal bridge (Table 3).

Poricalyptra aurisinae (Kamptner 1941) Kleijne 1991 (=Helladosphaera) (Fig. 4t), coccosphere ovoid (c. $9.0 \mu \mathrm{m})$ (Table 2). Coccoliths elliptical $(2.3-2.5 \mu \mathrm{m})$ (Table 3), with four oblong openings and transverse, virtually one-layered ridge on distal surface. 
Table 3 Comparison of the diameter/ length of body coccoliths of the twenty six first-time records in this study with those corresponding taxa reported by Cros \& Fortuño (2002) and Young et al. (2003)

\begin{tabular}{|c|c|c|c|}
\hline \multirow[t]{2}{*}{ Taxa } & \multicolumn{3}{|c|}{ Diameter/length of body coccolith $(\mu \mathrm{m})$} \\
\hline & This Study & Cros \& Fortuño (2002) & Young et al. (2003) \\
\hline Alisphaera pinnigera & $1.2-1.5$ & $1.5-1.6$ & $1.3-2.0$ \\
\hline Anacanthoica acanthos & $1.9-2.1$ & $2.1-2.6$ & n.d. \\
\hline Corisphaera gracilis & $1.2-1.5$ & $1.4-1.6$ & c. 1.5 \\
\hline Coronosphaera mediterranea $\mathrm{HOL}$ gracillima-type & $2.8-3.2$ & $2.1-2.3$ & n.d. \\
\hline Cyrtosphaera aculeata & $2.4-2.5$ & $2.5-2.8$ & $1.8-2.5$ \\
\hline Gephyrocapsa muellerae & $2.9-3.8$ & $3.1-3.9$ & $3-4$ \\
\hline Gephyrocapsa ornata & $2.8-3.6$ & $2.2-2.5$ & n.d. \\
\hline Helicosphaera carteri $\mathrm{HOL}$ & $2.4-2.7$ & $2.7-3.0$ & n.d. \\
\hline Helicosphaera hylina & $5.3-6.0$ & $6.2-6.8$ & n.d. \\
\hline Helicosphaera wallichii & $8.4-10$ & c. 9.0 & n.d. \\
\hline Holococcolithophora sphaeroidea & $1.5-2.0$ & $1.6-1.8$ & 1.8 \\
\hline Homozygosphaera arethusae & $1.7-2.1$ & $1.6-1.8$ & 1.5 \\
\hline Palusphaera vandelii & $1.2-1.8$ & $1.5-1.9$ & n.d. \\
\hline Papposphaera lepida & $1.2-1.6$ & $0.7-1.5$ & $1-1.5$ \\
\hline Polycrater galapagensis & $0.6-1.0$ & $0.6-0.7$ & n.d. \\
\hline Poricalyptra aurisinae & $2.3-2.5$ & $2.3-2.4$ & n.d. \\
\hline Rhabdosphaera xiphos & $1.3-1.9$ & $1.1-1.3$ & n.d. \\
\hline Reticulofenestra parvula & $1.9-2.8$ & $1.4-1.9$ & $1.2-2.0$ \\
\hline Reticulofenestra parvula var. tecticentrum & $2.5-3.0$ & $1.4-1.9$ & $1.2-2.0$ \\
\hline Syracosphaera anthos & $4.5-5.8$ & $2.2-2.5$ & $3.0-5.5$ \\
\hline Syracosphaera bannockii & $1.7-2.8$ & $1.5-1.7$ & n.d. \\
\hline Syracosphaera leptolepis & $1.8-2.0$ & $1.5-2.0$ & n.d. \\
\hline Syracosphaera nodosa & $1.3-2.7$ & $2.3-2.5$ & $1.5-2.5$ \\
\hline Syracosphaera nodosa aff. S. nodosa type 2 & $2.8-2.9$ & $2.7-2.9$ & n.d. \\
\hline Syracosphaera cf. orbiculus & $2.2-2.6$ & $2.0-2.2$ & $1.5-2.5$ \\
\hline Syracosphaera serrata & $2.1-2.2$ & n.d. & $1.5-2.5^{\mathrm{a}}$ \\
\hline
\end{tabular}

${ }^{a}$ Syracosphaera serrata [=S. nodosa type B, Young et al., 2003, p. 36, Plate 15]; n.d. = no da

Emiliania huxleyi (Lohmann 1902) Hay and Mohler in Hay et al. 1967 (Fig. 2c-g), small coccosphere (3.5$5.0 \mu \mathrm{m}$ ), spherical to subspherical, with relatively large elliptical coccoliths $(2.7-3.9 \mu \mathrm{m})$ (Table 2). Cells of the supercalcified and highly calcified forms more or less the same as other morphotypes; both forms recorded for the first time among Type A, B, and $\mathrm{C}$, in particular during the spring 2009 blooms east of New Zealand (Fig. 2c-e).

\section{Species diversity and distribution}

The total number of coccolithophores identified from samples collected between 2009 and 2012 was greatest to the east (46), intermediate to the west (along two transects in the Tasman Sea) (15), and least to the northeast (Bay of Plenty) and south (4 each) of New Zealand (Table 2). During the 3 year study two massive, almost monospecific, Emiliania huxleyi blooms were recorded in the vicinity of the STF (in the spring 2009 and summer 2011) and both were confirmed by the MODIS sensor on NASA's Aqua satellite (NASA Earth Observatory 2009, 2011). The total number of coccolithophores found on these two occasions was 4 and 6 taxa respectively.

In non-bloom conditions, based on the frequency of occurrence, Emiliania huxleyi was found to be most abundant at virtually all sampling stations. The only exception was in the autumn 2009, when Reticulofenestra parvula dominated the coccolithophore assemblage along the Kaikoura coast, east of New Zealand. In nonbloom conditions the average total number of taxa (22) recorded on five occasions, in the spring, summer and autumn of 2009, autumn 2011 and summer 2012, to the east, was greater than the average (7.6) recorded on three other occasions, in the summer 2010, spring 2009 and autumn 2011, to the rest of New Zealand (Table 2). 
The few members of the order Isochrysidales, e.g., Emiliania huxleyi, Gephyrocapsa ericsonii, G. muellerae and G. oceanica, were most widely distributed, being recorded at most sampling sites (Table 2). Even though members of the genus Syracosphaera generally occurred in low frequencies (e.g., S. pemmadiscus Chang 2013, S. bannockii, S. leptolepis, S. nana [Kamptner 1941] Okada and Mcintyre 1977 [=S. sp. type A of Kleijne 1991, 1993], S. nodosa, S. nodosa aff. S. sp. type 2, S. cf. orbiculus, S. ossa [Lecal 1966] Loeblich and Tappan 1968 [Syracolithus], S. serrata and S. tumularis Sánchez-Suárez 1990 [=S. sp. type C of Kleijne 1993]), they were found to be more widespread to the east (14) than to the west (4), northeast and south (none) of New Zealand (Table 2). All the remaining taxa appeared to be more sparsely distributed at fewer stations than the other two groups but generally they were also more common to the east than to the rest of New Zealand.

\section{Discussion}

The total of 46 living coccolithophores recorded here in the 3-year study is greater than the number previously documented (40) in New Zealand (Norris 1961; Cassie 1961; Burns 1977, Rhodes et al. 1993, 1994) and also is greater than the number (27) recently recorded on the three offshore stations, southeast of New Zealand (Saavedra-Pellitero et al. 2014). Nevertheless, more than half (c. $57 \%$ ) of these 46 taxa are first-time records. Adding 26 new records plus a recently described species, Syracosphaera pemmadiscus (Chang 2013) of this study, to the 40 previously documented taxa in New Zealand, and about 10 newly recorded taxa from southeast New Zealand, the total number of coccolithophores found in the New Zealand region is now estimated to be 77 . This is greater than the total of about 42 taxa recognised in the tropical, subtropical and temperate waters of Australia (about 16 are different from those recorded in the New Zealand region) (Conley 1979; Hallegraeff 1984, 2010; Callaghan 1992). By combining all the taxa identified in both New Zealand and Australia waters, the grand total of the current living coccolithophores in the southwest Pacific stands at about 93.

Most of the surveys (seven out of ten) conducted from 2009 to 2012 in this study were centred in the vicinity of the STF near $43 \mathrm{~S}$, east of New Zealand. This is a region of two contrasting water masses - warm, macronutrientpoor, iron-rich ST water in the north meeting cool, fresher, macronutrient-rich, iron-poor SA water in the south (e.g., Heath 1985; Chang and Gall 1998; Boyd et al. 1999) and is part of the so-called "The Great Calcite Belt" (Balch et al. 2011). The greater number of taxa plus two massive coccolithophore blooms (NASA Earth Observatory 2009, 2011) observed in the vicinity of STF compared with other areas of New Zealand, could reflect the wider seasonal range of sampling and greater number of surveys conducted near the STF. Nevertheless, it is likely that the physico-chemical conditions in this region favoured coccolithophores (e.g., Balch et al. 2011; Sadeghi et al. 2012; Saavedra-Pellitero et al. 2014).

In this study Emiliania huxleyi was virtually the only species observed to dominate in both bloom and nonbloom conditions. This observation is consistent with those made worldwide (e.g., Birkenes and Braarud 1952; Okada and Honjo 1975; Holligan et al. 1993; Cokacar et al. 2001; Wang et al. 2012; Saavedra-Pellitero et al. 2014). The build-up of Reticulofenestra parva as a dominant species in the spring 2009 off Kaikoura coast, however, was a unique event not just to New Zealand, but also in the southwest Pacific Ocean. Previously Reticulofenestra parva was only found to be dominant in surface sediments of the eastern Mediterranean Sea (Ziveri et al. 2000).

In terms of taxa, the genus Syracosphaera was the most diverse group. Fourteen taxa, about $31 \%$ of all extant coccolithophores, were recorded east of New Zealand alone. This number exceeded records of extant coccolithophores previously documented in New Zealand (12) (e.g., Cassie 1961; Norris 1961; Burns 1977; Saavedra-Pellitero et al. 2014), Australia (8) (Hallegraeff 1984, 2010) and also the Australian Sector of the Southern Ocean (9) (Findlay and Giraudeau 2000). With only a few exceptions, most of these Syracosphaera spp. occurred at low frequencies similar to reports elsewhere in the world (e.g., Kleijne 1993; Jordan and Kleijne 1994; Cros and Fortuño 2002; Young et al. 2003; Jordan et al. 2004).

\section{Conclusions}

Almost all 46 coccolithophores identified in this study were recorded from samples collected in the vicinity of Chatham Rise and Kaikoura coast, east of New Zealand alone. In contrast, a relatively small number of coccolithophores were recorded from samples collected to the west (in the Tasman Sea), northeast (Bay of Plenty) and south of the country. About $57 \%$ of these taxa/ forms are first-time records. Emiliania huxleyi was the dominant species observed not only in the two almost monospecific blooms east of New Zealand, but also in most cases, in non-bloom conditions. Reticulofenestra parva was, however, found on one occasion, to be dominant off Kaikoura coast. Even though Syracosphaera taxa generally occurred at low frequencies, they were the largest group and made up c. $31 \%$ of all extant coccolithophores recorded in this study. The total number of coccolithophores currently estimated in the Southwest Pacific near New Zealand and Australia is 93.

\section{Abbreviations}

aff., species affinis or akin to; $c f$., see also; HOL, holococcolithophore; MODIS, Moderate Resolution Imaging Spectroradiometer; var., variety 


\section{Acknowledgments}

We thank Dr Janet Grieve of NIWA, Wellington, for her constructive criticisms of this manuscript, Dr Cliff Law for initiating and coordinating the coccolithophore surveys, and last but not least, Dr Annelies Kleijne of Geomarine Centre, Vrije Universiteit, The Netherland, and Dr Luïsa Cros of Institut de Ciéncies del Mar (CSIC), for the clarification of the taxonomy of several Syracosphaera taxa. The assistance of the crew and officers of the NIWA Research Vessels Tangaroa and Kaharoa during all surveys is gratefully acknowledged. Funding from the New Zealand Ministry for Primary Industries and from NIWA under Coasts and Oceans Research Programme 2 (2013/14 SCl) provided support for this work.

\section{Availability of data and material}

Not applicable.

\section{Authors' contributions}

FHC: conceived of the study, conducted the SEM examination, identified all the taxa of extant coccolithophores and drafted the manuscript. LN: collected the samples from each voyage. Both authors read and approved the final manuscript.

\section{Competing interests}

The authors declare that they have no competing interests.

\section{Consent for publication}

Not applicable.

\section{Ethics approval and consent to participate}

Not applicable.

\section{Author details}

'Biodiversity and Biosecurity Group, National Institute of Water \& Atmospheric Research Ltd., P. O. Box 14-901, Kilbirnie, Wellington 6241, New Zealand. ${ }^{2}$ Ocean Sediments Group, National Institute of Water \& Atmospheric Research Ltd., P. O. Box 14-901, Kilbirnie, Wellington 6241, New Zealand.

\section{Received: 26 July 2016 Accepted: 26 July 2016}

\section{Published online: 15 August 2016}

\section{References}

Balch WM, Drapeau DT, Bowler BC, Lyczskowski E, Booth S, Alley D. The contribution of coccolithophores to the optical and inorganic carbon budgets during the Southern Ocean Gas Exchange Experiments: New evidence in support of the Great Calcite Belt hypothesis. J Geophys Res. 2011;116:COOF06. doi:10.1029/2011JC006941.

Baumann K-H, Andruleit HA, Samtleben C. Coccolithophores in the Nordic Seas: comparison of living communities with surface sediment assemblages. Deep-Sea Res II. 2000:47:1743-72.

Beaufort L, Probert I, De Garode-Thoron T, Bendif EM, Ruiz-Pino D, Metzl N, Goyet C, Buchet N, Coupel P, Grelaud M, Rost B, Rickaby REM, De Vargas C. Sensitivity of coccolithophores to carbonate chemistry and ocean acidification. Nature. 2011; 476:80-3.

Biekart JW. The distribution of calcareous nannoplankton in late Quaternary sediments collected by the Snellius II Expedition in some southeast Indonesian basins. Proceedings Koninklijke Nederlandse Akadem van Wetenschappen, B, 1989;92:77-141.

Birkenes E, Braarud T. Phytoplankton in the Oslo Fjord during a "Coccolithus huxleyi - summer". Avhabdkubger ytgutt av det Norske Videnskap- Akademi Oslo (Matem Naturvid Klasse); 1952. p. 1-23.

Borsetti AM, Cati F. II nannoplancton calcareo vivente nel Tirreno centromeridionale. Parte II. Giornale Geol. 1976;40:209-40.

Boyd PD, LaRoche J, Gall M, Frew R, McKay RML. The role of iron, light and silicate in controlling algal biomass in sub-Antarctic water SE of New Zealand. J Geophys Res. 1999;104(C6):13395-408.

Bréhéret JG. Formes nouvelles quaternaries et actualles de la famille des Gephyrocapsaceae (Coccolithophorides). C R Acad Sci, Paris Série D. 1978;287:447-9.

Burns DA. The latitudinal distribution and significance of calcareous nannofossils in the bottom sediments of the South-west Pacific Ocean (Lat. 15-55็s) around New Zealand. In: Fraser R, editor. Oceanography of the South Pacific for UNESCO, Wellington; 1973. p. 221-8.
Burns DA. The abundance and species composition of nannofossil assemblages in sediments from continental shelf to offshore basin, western Tasman Sea. Deep-Sea Res. 1975;22:425-31.

Burns DA. Phenotypes and dissolution morphotypes of the genus Gephyrocapsa Kamptner and Emiliania huxleyi Lohmann. New Zealand I Geol Geophys. 1977;20:143-55.

Callaghan KM. A taxonomic survey of the coccolithophorids of Bass Strait, with life history observations. B.Sc. (Hons.) thesis, University of Melbourne; 1992.

Cassie V. Marine phytoplankton in New Zealand waters. Bot Mar. 1961;2(Suppl):1-54.

Chang FH. Syracosphaera pemmadiscus sp. nov. (Prymnesiophyceae), an extant coccolithophore from the southewest Pacific Ocean near New Zealand. Phycologia. 2013;52(6):618-24.

Chang FH, Gall M. Phytoplankton assemblages and photosynthetic pigments during winter and spring in the Subtropical Convergence region near New Zealand. New Zealand J Mar Freshw Res. 1998;32:515-30.

Cokacar T, Kubilay N, Oguz T. Structure of Emiliania huxleyi blooms in the Black Sea surface waters as detected by SeaWiFS imagery. Geophys Res Lett. 2001; 28:4607-10.

Conley SM. Recent coccolithophores from the Great Barrier Reef-Coral Sea region. Micropaleontology. 1979;25:20-40.

Cros L, Kleijne A, Zeltner A, Billar C, Young JR. New examples of holococcolithheterococcolith combination coccospheres and their implications for coccolithophorid biology. Mar Micropaleontol. 2000;39:1-34.

Cros L, Fortuño J-M. Atlas of Northwestern Mediterranean Coccolithophores. Sci Mar. 2002;66 Suppl 1:7-182.

Deflandre G. Glasse des coccolithophoridés. (Coccolithophoridae Lohmann, 1902). In: Grassé P-P, editor. Traité de Zoologie 1. Paris: Masson; 1952. p. 439-70.

Deflandre G, Fert C. Observations sur les coccolithophoridés actuels et fossils en microscopie ordinaire et électronique. Ann de Paléontol. 1954;40:115-76.

Doney SC, Fabry VJ, Feely RA, Kleypas JA. Ocean acidification: the other $\mathrm{CO}_{2}$ problem. Ann Rev Mar Sci. 2009;1:169-92.

Edwards AR. The calcareous nannoplankton - evidence for New Zealand Tertiary marine climate. Tuatara. 1968;16:26-31.

Edwards AR. Calcareous nannofossils. In: Hoskins RH, editor. Stages of the New Zealand Cenozoic: a synopsis, New Zealand Geol Surv Rep, vol. 107. 1982. p. 23-7.

Eynaud F, Giraudeau J, Pichon JJ, Pudsey CJ. Sea surface distribution of coccolithophores, diatoms, silicoflagellates and dinoflagellates in the South Atlantic Ocean during the late austral summer 1995. Deep-Sea Res Part I. 1999:46:451-82.

Findlay CS, Giraudeau J. Extant calcareous nannoplankton in the Australian Sector of the Southern Ocean (Austral summers 1994 and 1995). Mar Micropaleontol. 2000;40:417-39.

Findlay CS, Young JR, Scott FJ. 6. Haptophytes: order Coccolithopharales. In: Scott FJ, Marchant HJ, editors. Antarctic marine protists. Canberra: Australian Biological Resource Study; 2005. p. 276-94.

Frada M, Young J, Cacháo M, Lino S, Marcosp A, Probert I, Vargas CD. A guide to extant coccolithophores (Calcihaptophycidae, Haptophyta) using light microscopy. J Nannoplankton Res. 2010;31:58-112.

Gaarder KR. Three New Taxa of Coccolithineae. Nytt Mag Bot. 1970;17:113-26.

Hallegraeff GM. Coccolithophorids (Calcareous nanoplankton) from Australian Waters. Bot Mar. 1984;27:229-47.

Hallegraeff GM. Coccolithophorids (Haptophyta). In: Hallegraeff GM, Bolch CJS, Hill DRA, Jameson I, LeRoi J-M, Murray S, De Salas MF, Saunders K, editors. Algae of Australia phytoplankton of temperate coastal waters. Department of the Environment, Water, Heritage and the Arts, CSIRO Publishing; 2010. p. 342-60.

Hay WW. Carbonate fluxes and calcareous nannoplankton. In: Thierstein HR Young JR, editors. Coccolithophores: from molecular processes to global impact. Singinger-Verlag Berlin Heidelberg; 2004. p. 509-29.

Hay WW, Mohler HP, Roth PH, Schmidt RR, Boudreaux JE. Calcareous nanoplankton zonation of the Cenozoic of the Gulf Coast and CaribbeanAntillean area, and transoceanic correlation. Trans Gulf Coast Assoc Geol Soc. 1967; $17: 428-80$.

Heath RA. Large-scale influence of the New Zealand seafloor topography on western boundary currents of the South Pacific Ocean. Aust J Mar Freshw Res. 1985;36:1-14.

Heimdal BR. Two new taxa of recent coccolithophorids. "Meteor" Forsch-Ergebn, Reihe D, Biol. 1973;13:70-5.

Heimdal BR, Gaarder KR. Coccolithophorids from the northern part of the eastern central Atlantic I. Holococcolithophorids. "Meteor" Forsch-Ergebn, Reihe D, Biol. 1980;32:1-14. 
Hiramatsu C, De Deckker P. Distribution of calcareous nannoplankton near the Subtropical Convergence, south of Tasmania, Australia. Aust J Mar Fresh Res. 1997:47:707-13

Holligan PM, Fernández E, Aiken J, Balch WM, Boyd P, Burkill PH, Finch M, Groom SB, Malin G, Muller K, Purdie DA, Robinson C, Trees CC, Turner SM, van der Wal P. A biogeochemical study of the coccolithophore, Emiliania huxleyi, in the North Atlantic. Global Biogeochem Cycles. 1993;7(4):879-900.

Janin M-C. Micropaléontologie de concretions polymétaliques du Pacifique central: zone Clarion-Clipperton, chaine Centre-Pacifique, lles de la Ligne et archipel des Tuoamotou (Eocéne-Actuel). Mém Soc Géol Fr. 1987;152:1-317.

Jordan RW, Young JR. Proposed changes to the classification system of living coccolithophorids. Int Nannoplankton Assoc Newslett. 1990;12:15-8.

Jordan RW, Kleijne A. A classification system for living coccolithophores. In: Winter A, Siesser WG, editors. Coccolithophores. Cambridge University Press; 1994. p. 83-105.

Jordan RW, Cros L, Young JR. A revised classification scheme for living haptophytes. In: Triantaphyllou MV, editor. Advances in biology, ecology and taxonomy of extant calcareous nannoplankton. New York: Micropaleontology Press, Micropaleontology 2004;50 Suppl 1:55-79.

Kamptner E. Neue und bemerkenswerte Coccolithineen aus dem Mittelmeer. Arch Protistenk. 1937;89:279-316.

Kamptner E. Die Coccolithineen der Südwestküste von Istrien. Ann Naturh Mus Wien. 1941;51:54-149.

Kamptner E. Zur Revision der Coccolithineen-Spezies Pontosphaera huxleyi Lohm. Akad Wiss Wien, Math-Naturw Kl. 1943:80:43-9.

Kamptner E. Untersuchungen über den Feinbau der Coccolithen. Arch Protistenk. 1954;100:1-90.

Kleijne A. Holococcolithophorids from the Indian Ocean, Red Sea, Mediterranean Sea and North Atlantic Ocean. Mar Micropaleontol. 1991;17:1-76.

Kleijne A. Extant Rhabdosphaeraceae (coccolithophorids, Class Prymnesiophyceae) from the Indian Ocean, Red Sea, Mediterranean Sea and North Atlantic Ocean. Scripta Geol. 1992;100:1-61.

Kleijne A. Morphology, taxonomy and distribution of extant coccolithophorids (calcareous nannoplankton). Drukkerij FEBO B.V.; 1993. 321 pp.

Kleijne A, Jordan RW, Heimdal BR, Samtleben C, Chamberlain AHL, Cros L. Five new species of the coccolithophorid genus Alisphaera (Haptophyta), with notes on their distribution, coccolith structure and taxonomy. Phycologia. 2002;40:583-601.

Kleijne A, Cros L. Ten new extant species of the coccolithophore Syracosphaera and a revised classification scheme for the genus. Micropaleontology. 2009; 55:425-62.

Lecal J. Un nouvel Hymenomonas: H. prenantii n. sp. (Cocccolithophoridés). Ann Limnol. 1965;1:155-62.

Lecal J. Coccolithophorides littoraux de Banyuls. Vie et Millieu. 1966;16:251-70.

Loeblich AR, Tappan H. Annotated index and bibliography of the calcareous nannoplankton II. J Paleontol. 1968;42:584-98.

Lohmann H. Die coccolithophoridae, eine monographic der coccolithen bildenden flagellaten. Zugleich ein Beitrag zur Kenntnis des Mitterlmeerauftriebs. Arch Protistenk. 1902;1:89-165.

Lohmann $\mathrm{H}$. Untersuchungen über das Pflanzen- und Tierleben der Hochsee, zugleich ein Bericht über die biologischen Arbeiten auf der Fahrt der "Deutschland" von Bremerhaven nach Buenos-Aires in der Zeit vom 7, Mai bis 7. September 1911. Univ Berlin, Veröff Inst Meereschundund. GeograpNat wiss. 1912;1:1-92.

Manton I, Leadale GF. Observations on the microanatomy of Coccolithus pelagicus and Cricosphaera carterae, with special reference to the origin and nature of coccoliths and scales. J Mar biol Ass UK. 1969;49:1-16.

Manton I, Oates K. Polycrater galapagensis gen. et sp. nov., a putative coccolithophorid from the Galapagos Islands with an unusual aragonitic periplast. Br Phycol J. 1980;15:95-103.

McIntyre A, Bé AWH. Modern coccolithophoridae of the Atlantic Ocean - I. Placoliths and cyrtholiths Deep-Sea Res. 1967;14:561-9.

Mclntyre A, Bé AWH, Roche B. Modern Pacific coccolithophorida: a paleontological thermometer. New York: Academic of Science. Trans Series II. 1970;32(6):720-31.

Murray J, Renard AF. Report on the Scientific Results of the Voyage of H.M.S. Challenger during the year 1873-76. Deep-Sea Deposits. HMSO, London xxxiv; 1891. + 525 p., 29 pls., 43 charts, 22 diagrams.

NASA. Earth Observatory. In: earthobservatory.nasa.gov/IOTD/view.php?id = 40924; 2009.
NASA Earth Observatory. In: earthobservatory.nasa.gov/IOTD/view.php?id= 49459; 2011.

Nishida S. Nannoplankton flora in the Southern Ocean, with special reference to siliceous varieties. Mem Nat Inst Polar Res, Spec Issue. 1986;40:56-68.

Norris RE. Observations on phytoplankton organisms collected on the N.Z.O.I. Pacific Cruise, September 1958. N Z J Sci. 1961;4:162-88.

Norris RE. Indian Ocean nannoplankton. I. Rhabdosphaeraceae (Prymnesiophyceae) with a review of extant taxa. J Phycol. 1984;20:27-41.

Okada H, Honjo S. Distribution of coccolithophores in Marginal Seas along the Western Pacific Ocean and in the Red Sea. Mar Biol. 1975;31:271-85.

Okada H, McIntyre A. Modern coccolithophores of the Pacific and north Atlantic Oceans. Micropaleontology. 1977;23:1-55.

Probert I, Fresnel J, Billard C, Geisen M, Young JR. Light and electron microscope observation of Algirosphaera robusta (Prymnesophyceae). J Phycol. 2007;43: 319-32.

Rhodes LL, Haywood A, Ballantine WJ, MacKenzie AL. Algal blooms and climate anomalies in north-east New Zealand, August- December 1992. N Z J Mar Freshw Res. 1993;27:419-30.

Rhodes LL, Peake B, MacKenzie AL, Marwick S. Coccolithophores Gephyrocapsa oceanica and Emiliania huxleyi (Prymnesiophyceae $=$ Haptophyceae) in New Zealand's coastal waters: Characteristics of blooms and growth in laboratory culture. N Z J Mar Freshw Res. 1994;29:345-57.

Rowson JD, Leabeater BSC, Green JC. Calcium carbonate deposition in the motile (Crystallolithus) phase of Coccolithus pelagicus (Prymnesiophyceae). Br Phycol J. 1986;21:359-70.

Ruggiero MA, Gordon DP, Orrell TM, Bailly N, Bourgoin T, Brusca RC, GavalierSmith T, Guiry MD, Kirk PM. A higher level classification of all living organisms. Plos One 2015; p 60. DOI: 10.1371

Sánchez-Suárez IG. Three new Coccolithophorids (Haptophyta) from the SouthEastern Caribean Sea: Cyclolithellla ferrazae sp. nov. Syracosphaera florida sp. nov. Syracosphaera tumularis sp. nov. Biol Mar Acta Cientifica Venezolana. 1990;41:152-8.

Saavedra-Pellitero M, Baumann K-L, Flores J-A, Gersonde R. Biogeographic distribution of living coccolithophores in the Pacific sector of the Southern Ocean. Mar Micropaleontol. 2014;109:1-20.

Sadeghi A, Dinter T, Gountas M, Taylor B, Altenburg-Soppa M, Bracher A. Remote sensing of coccolithophore blooms in selected oceanic regions using the PhytoDOAS method applied to hyper-spectral satellite data. Biogeosciences. 2012;9:2127-43.

Schiller J. Vorlãufige Ergebnisse der Phytoplankton-Untersuchungen auf den Fahrten S.M.S. Najade in der Adria 1911/12. I. Die Coccolithophoriden- K Akad Wiss, Wien, Sitzber, Math Naturw Klasse. 1913;122(1):597-617

Schiller J. Die planktonischen vegetationen des adriatischen Meeres. A. Die coccolithophoriden-Vegetation in den Jahren 1911-14. Arch Potistenk. 1925; 51:1-130.

Tangen K. Papposphaera lepida, gen. nov. sp., a new marine coccolithophorid from Norwegian coastal waters. Nor J Bot. 1972;19:171-8.

Thomsen HA. Identification by electron-microscopy of nannoplanktonic coccolithophorids (Prymnesiophyceae) from West Greenland, including the description of Papposphaera sarion sp. nov. Br Phycol J. 1981;16(1):77-94.

Tyrrell T, Merico A. Emiliania huxleyi: bloom observations and the conditions that induce them. In: Thierstein HR, Young JR, editors. Coccolithophores: from molecular processes to global Impact. Singinger-Verlag Berlin Heidelberg; 2004. p.75-97.

Wallich GC. Observations on the coccosphere. Ann Magaz Nat Hist. 1877;9:342-50.

Wang J, Luan Q, Zuo T, Chen R, Sun J. Taxonomic composition of marine-living cocolithophores in the Yellow Sea and East China Sea - new records and a species list. Mar Biodivers Rec. 2012;5:1-8.

Winter A, Siesser WG. Atlas of living coccolithophores. In Winter A, Siesser WG, editors. Coccolithophores. Cambridge Unversity Press; 1994. p. 107-59.

Young JR, Geisen M, Cros L, Kleijne A, Sprengel C, Probert I, Østergaard J. A guide to extant coccolithophore taxonomy. J Nannoplankton Res Spec Issue. 2003;1:125.

Ziveri P, Rutten A, de Lange GJ, Thomson J, Corselli C. Present-day coccolith fluxes recorded in central eastern Mediterranean sediment traps and surface sediments. Palaeogeog Palaeoclimatol Palaeoecol. 2000;158:175-95. 